\title{
Fast-Painting of Human Metaphase Spreads Using a Chromosome-Specific, Repeat-Depleted DNA Library Probe
}

BioTechniques 24:820-825 (May 1998)

\begin{abstract}
M. Durm, L. Schüssler, H. Münch, J. Craig', H. Ludwig, M. Hausmann and C. Cremer University of Heidelberg, Heidelberg, and ${ }^{1}$ University of Munich, Munich, Germany
\end{abstract}

\section{ABSTRACT}

For chromosome painting, in situ suppression of repetitive DNA sequences has been well established. Such standard protocols usually require large amounts of Cot-1 $D N A{ }^{\circledR}$. Recently, it has become possible to deplete repetitive DNA sequences from library probes by magnetic purification and PCR-assisted affinity chromatography. These "repeat-depleted library probes" appear to be extremely useful for Fast-FISH, a technique that omits denaturing chemical agents such as formamide in the hybridization buffer, resulting in a substantial acceleration and simplification of the complete protocol. Shown here is the application of Fast-FISH to a repeat-depleted, directly fluorochrome-labeled library probe of the qarm of chromosome 15 (Fast-Painting) for human lymphocyte metaphase spreads. Following painting without Cot-1 DNA and without formamide, visual inspection revealed sufficient chromosome painting after a few hours of hybridization. The fluorescence signals of the labeling sites were analyzed after hybridization times of 1 and $2 \mathrm{~h}$ (in one case, 4 h) using digital fluorescence microscopy. The painting efficiency expressed in values of relative fluorescence signal ratios was quantitatively evaluated by image analysis using line-scan procedures and area-morphometry of mean luminance. Two preparation protocols (ethanol dehydration without and with RNase A treatment followed by pepsin digestion for four different exposure times) were compared. These results indicated that RNase A treatment and pepsin digestion are steps that can be omitted.

\section{INTRODUCTION}

Standard protocols of fluorescence in situ hybridization (FISH) to specifically label metaphase chromosomes or chromatin targets in cell nuclei intensively use denaturing chemical agents, especially formamide (for review see Reference 12), in concentrations of $50 \%-70 \%$ ( $\mathrm{vol} / \mathrm{vol})$. In most cases, the hybridization time is extremely prolonged, and repeated attention is required for the many washing steps prescribed. In the case of chromosome painting (15), specifically, chromosome in situ suppression (CISS) hybridization $(4,13)$, a pre-annealing procedure for in situ suppression of repetitive DNA sequences is additionally required. This can be done for instance by using Cot- $1 \mathrm{DNA}^{\circledR}$ in high concentrations.

Recently, a new FISH technique, called Fast-FISH $(2,6,10,11)$, has been developed. This technique omits the use of formamide or other equivalent denaturing chemical agents. For several centromere-specific DNA probes (e.g., chromosome 1, 8, 12, 15 and $\mathrm{X}$ ), the hybridization time has been reduced to typically $60 \mathrm{~min}$, resulting in a complete FISH procedure of $3 \mathrm{~h}$ maximum, including indirect fluorochrome labeling.

Although Fast-FISH is working with high reproducibility for repetitive DNA probes, it appeared difficult to adapt the principle and its protocol to chromosome-painting probes (7). A major reason for such difficulties might have been the presence of probe sequences specific for ubiquitous repetitive DNA sequences in the chromosomal targets.

Recently, it has become possible to deplete repetitive DNA sequences from DNA-library probes (3). Such "repeatdepleted library probes" are ideally suitable for a Fast-FISH painting procedure. In this paper, we present FastPainting results for a repeat-depleted DNA library probe of human chromosome $15 q$. This library was constructed (3) from a microdissection chromosome arm library of $15 \mathrm{q}$ (9) (kindly provided by Drs. J. Trent and M. Bittner, NIH, Bethesda). Besides the physical parameters of hybridization time and temperature, additional effects of often-applied target treatment protocols (RNase treatment and pepsin digestion) were analyzed. Signal intensity, speci- 
ficity and signal ratios were quantitatively analyzed by digital-image procedures after acquisition of fluorescence microscopy images by a cooled truecolor, charge-coupled device (CCD) camera.

\section{MATERIALS AND METHODS}

\section{Slide Preparation}

Metaphase chromosomes were obtained from human lymphocytes isolated from peripheral blood (from adults) by standard techniques (1). The lymphocytes were stimulated by phytohemagglutinin $\mathrm{M}(2.5 \mu \mathrm{L} / \mathrm{mL}$ lymphocyte medium; Boehringer Mannheim GmbH, Mannheim, Germany) and cultivated for $72 \mathrm{~h}$ (chromosomenmedium B; Biochrom KG, Berlin, Germany) followed by a Colcemid ${ }^{\circledR}$ block (Boehringer Mannheim) for 2 additional hours. Metaphase spreads on slides were obtained after a hypotonic shock with $\mathrm{KCl}(0.075 \mathrm{M})$ at $37^{\circ} \mathrm{C}$ and methanol/acetic acid (3:1, vol/vol) fixation (14). Before hybridization, the slides with metaphase preparations were fur- ther treated in two different ways:

(i) The slides were dehydrated by ethanol, first at $70 \%$ overnight, then stored in $100 \%$ ethanol.

(ii) The slides were dehydrated by an ethanol series of increasing percentage $(70 \%, 85 \%$ and $100 \%)$ after an RNase A treatment $(1 \mathrm{mg} / \mathrm{mL}$ of RNase A; Sigma-Aldrich Chemie $\mathrm{GmbH}$, Deisenhofen, Germany, in $2 \times$ standard saline citrate $\left[\mathrm{SSC}\right.$ ] for $1 \mathrm{~h}$ at $37^{\circ} \mathrm{C}$ ) followed by a pepsin digestion $(0.005 \%$ in $\mathrm{HCl} 0.01 \mathrm{~N}$ for 5, 10, 20 and $40 \mathrm{~min}$ at $37^{\circ} \mathrm{C}$ ) and an incubation in a postfixation mixture $\left(\mathrm{MgCl}_{2} 0.05 \mathrm{M}\right.$, formaldehyde $0.1 \%$ in phosphatebuffered saline [PBS], $\mathrm{pH}$ 7.0) for 10 $\mathrm{min}$ at room temperature. All the different steps were separated by washing in $0.9 \% \mathrm{NaCl}$ or $2 \times \mathrm{SSC}$ for $5 \mathrm{~min}$.

\section{In Situ Hybridization}

The repeat-depleted DNA library probe [the depletion procedure was performed as described in detail elsewhere (3)] was labeled with fluorescein-11-dUTP using standard nick translation procedures (Nick Translation Kit; Boehringer Mannheim

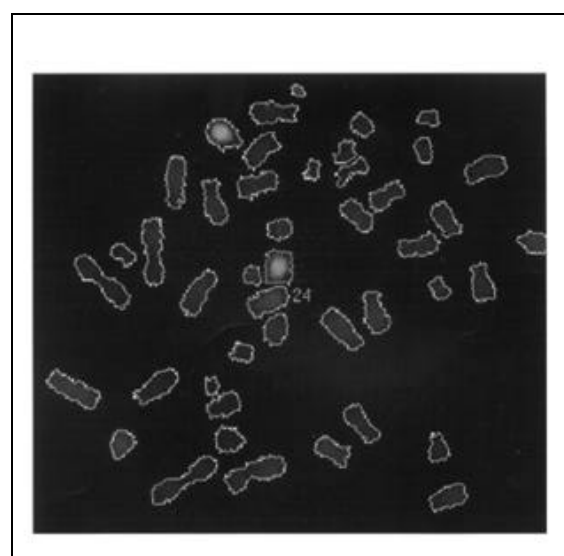

a

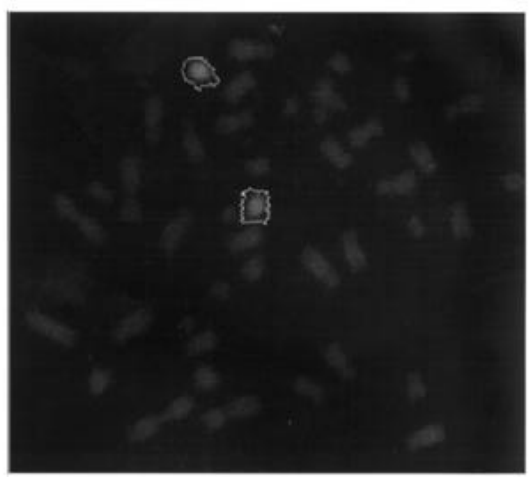

b

Figure 1. (a) Digitized image of a metaphase spread following Fast-Painting. As a first step of image analysis, all chromosomes of the metaphase spread were segmented using an ideal global threshold. The segmented chromosome areas are outlined by a white edging. As an example, the number indicates a chromosome selected for data extraction. This number is arbitrary and simply indicates the position of the segmented chromosome within the image. In a second evaluation step, the three brightest chromosomes (i.e., the three chromosomes with the highest mean luminance) were detected and used for the further analysis applied here (see Figure 3 and 4). (b) In the same metaphase spread as in Panel a, the painted chromosomes were automatically separated from the non-painted ones using a threshold $\mathrm{T}_{\mathrm{c}}$ for the mean luminance intensity of each segmented chromosome. The required threshold $\mathrm{T}_{\mathrm{c}}$ was automatically determined to be $60 \%$ of the mean luminance of the brightest chromosome in the metaphase spread. In this case, as expected, two chromosomes with a mean luminance intensity above the threshold $\mathrm{T}_{\mathrm{c}}$ were found.

$\mathrm{GmbH})$. Approximately $250 \mathrm{ng}$ of the labeled DNA probe, $1 \mu \mathrm{L}$ hybridization buffer (10×: $100 \mathrm{mmol} / \mathrm{L}$ Tris-HCl, 30 $\mathrm{mmol} / \mathrm{L} \mathrm{MgCl}_{2}, 500 \mathrm{mmol} / \mathrm{L} \mathrm{KCl}, \mathrm{pH}$ 8.3) and $1 \mu \mathrm{L} 20 \times$ SSC were diluted in a solution of dextran sulfate $(10 \%$ in the final volume, Av. Mol. Wt. 500000; Sigma-Aldrich Chemie $\mathrm{GmbH}$ ) in deionized $\mathrm{H}_{2} \mathrm{O}$ to make up a final volume of $10 \mu \mathrm{L}$. This hybridization mixture was pipetted on the microscope slides with the fixed metaphase spreads. The slides were covered with a $18-\times 18-\mathrm{mm}$ cover glass, sealed with rubber cement (Fixogum; Marabu, Tamm, Germany) and placed in a specially designed, closed stainless steel chamber. Thermal denaturation was performed at $94^{\circ} \mathrm{C}$ for $3 \mathrm{~min}$. For hybridization, the steel chamber with the slides was placed into a water bath of $70^{\circ} \mathrm{C}$ for 1,2 or $4 \mathrm{~h}$. Then the slides were washed once at $37^{\circ} \mathrm{C}$ for $10 \mathrm{~min}$ in physiological $\mathrm{NaCl}$ solution containing $0.02 \%$ Tween $^{\circledR} 20$. For counterstaining of the chromosomes, the slides were mounted in VECTASHIELD ${ }^{\mathrm{TM}}$ Antifade Mounting Medium (Vector Laboratories, Burlingame, CA, USA) containing $5 \mu \mathrm{mol} / \mathrm{L}^{\prime}$,6-diamidino-2phenylindole (DAPI).

\section{Microscopy and Digital Image Analysis}

For visualization, an Orthoplan Fluorescence Microscope (Leica, Wetzlar, Germany) was used equipped with a PlanAPO 63×/NA 1.40 objective and a $100-\mathrm{W}$ mercury arc lamp. Excitation and detection took place by use of a triple-band-pass filter-set (Model 506 D/4C/10C; Chroma Technology, Brattleboro, VT, USA) appropriate for simultaneous DAPI, fluorescein isothiocyanate (FITC), tetramethylrhodamine isothiocyanate (TRITC) excitation and detection. On the slides, metaphase spreads were chosen by random access, and their fluorescence images were registered by a true-color CCD camera (Model CF 20 DXC; Kappa, Gleichen, Germany) with an interline CCD chip with integrated mosaic filter and a resolution of $752 \times 582$ pixels. This camera digitizes the recorded images directly, omitting a frame-grabber board in the computer. For registration and interactive evaluation, the commercially 
available software package OPTIMASTM $^{\mathrm{TM}}$ (Optimas, Bothell, WA, USA) was running on a $\mathrm{PC}\left(\right.$ Pentium $\left.^{\circledR}\right)$ under WINDOWS $95^{\circledR}$ (Microsoft, Redmond, WA, USA). This software package allows the direct implementation of program subroutines designed in our laboratory specifically for the evaluations presented here.

The digitized raw images were visualized and evaluated without any further processing, such as filtering, contrastenhancing operations or background corrections. All quantitative results were obtained from the green image plane ("green light" contribution to the real color image) using a sequence of OPTIMAS subroutines and $\mathrm{C} / \mathrm{C}++-$ DLLs (Dynamic Link Library) controlled by a windows dialogue. This windows dialogue allows the automatic segmentation of the chromosomes by an optimized global threshold (Figure 1a):

Using a weighted gray value histogram of the green image plane, every possible threshold value $\mathrm{T}$ was tested by an algorithm (kindly obtained from Dr. Joachim Dengler, German Cancer Research Center, Heidelberg) that determined the best threshold value, which was then used for segmentation. Segmented chromosomes that were attached to each other were separated interactively. To distinguish between labeled and nonlabeled chromosomes, the mean luminance (defined here as the mean gray value of the green image plane) of the segmented chromosome areas was calculated, and the threshold was determined. The required threshold $\mathrm{T}_{\mathrm{c}}$ was automatically determined in each metaphase spread as $60 \%$ of the mean luminance of the brightest chromosome (Figure 1b).

To measure the FISH efficiency on the basis of signal-to-noise ratio, in each metaphase spread, the data from the three chromosomes with the brightest mean luminance (i.e., the two labeled and the brightest nonlabeled chromosome) were considered for statistical evaluation. Two different procedures were applied: (i) a classical standard method (line scan) and (ii) a new method that fits better in the present case (mean luminance scan).

Line scan. To register intensity profiles and maximum values, a line scan with a width corresponding to 10 pixels was interactively performed along the main chromosome axis of the three brightest chromosomes, which were automatically determined. For background subtraction, the minimum value of the line scan observed along each chromosome was used, and, for each profile, the maximum intensity value

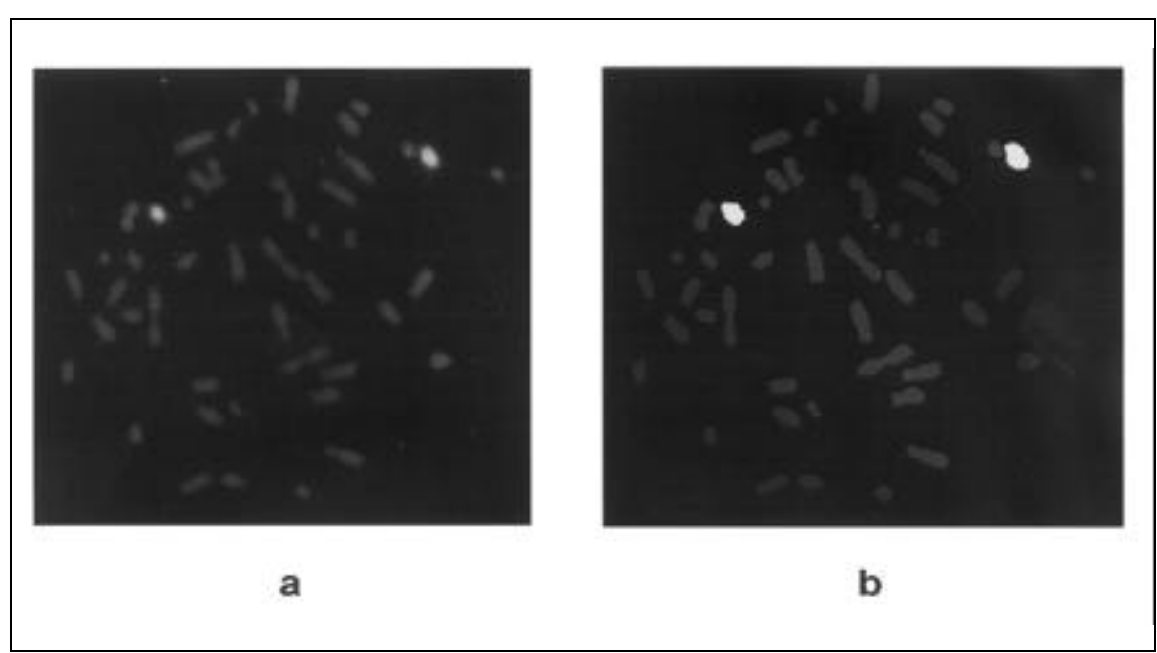

Figure 2. Example of a human metaphase spread with the two homologous 15q chromosome arms labeled by Fast-Painting. The repeat-depleted DNA painting probe of $15 \mathrm{q}$ was directly labeled with FITC. For counterstaining, DAPI in VECTASHIELD mounting medium was used. The slides were dehydrated by an ethanol series. No RNase treatment and no pepsin digestion were used in this case. The hybridization time was $2 \mathrm{~h}$. Panel a shows the raw data CCD image, whereas Panel $\mathrm{b}$ shows the same image after an automatic global segmentation and discrimination. The automatic evaluation (compare Figure 1) confirmed the visual inspection.

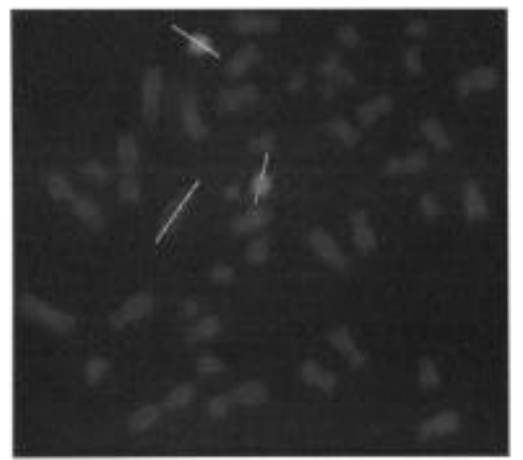

a

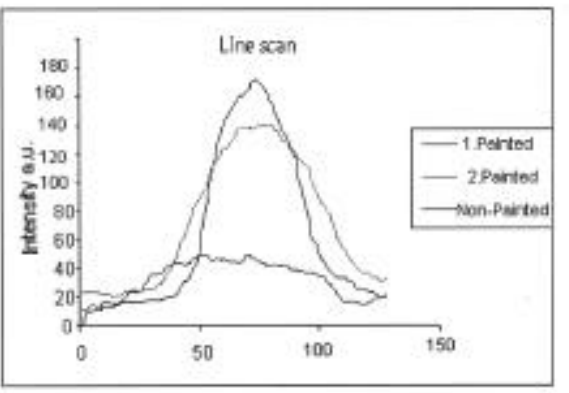

b
Figure 3. (a) A line scan with a width corresponding to 10 pixels (about $2 / 3$ of the chromosome width) was interactively performed along the main chromosome axis of the two painted chromosomes of the metaphase spread shown in Figure 1, a and b, and the brightest non-painted chromosome in the green image plane. These chromosomes were chosen according to their automatically determined mean luminance. After a background correction, using the minimum value of the line scans from each profile, the maximum intensity was extracted. The image shows a metaphase spread after Fast-Painting with 2-h hybridization time and without RNase/pepsin treatment (camera exposure time of $160 \mathrm{~ms}$ without electronical enhancement). (b) Result of the line scans (averaged intensity [a.u.] perpendicular to the chromosome axis vs. arbitrary units along the chromosome axis). The two painted chromosomes show a considerable intensity increase on the long chromosome arm. 
number of individual metaphase spreads and then averaged to determine a FISH-efficiency factor for each specimen protocol modification. The data obtained were further processed by a spreadsheet program.

Mean luminance scan. From the computed mean luminance of the segmented chromosomes of each metaphase spread, the ratio of the mean values of the two brightest chromosomes (i.e., the painted chromosomes) to the brightest non-painted chromosome was calculated. These values were again determinded for a number of metaphase spreads and then averaged to determine the FISH-efficiency factor for each specimen protocol modification.

The data were further processed by a spreadsheet program.

\section{RESULTS}

Fast-Painting of human lymphocyte metaphase spreads was performed using a degenerate oligonucleotide-primed (DOP)-polymerase chain reaction (PCR)-amplified, repeat-depleted, directly labeled library probe of the q-arm of chromosome 15 . This probe was applied without any pre-annealing steps in the hybridization procedure. The in situ hybridization itself was performed at $70^{\circ} \mathrm{C}$ without formamide and without Cot-1 DNA. The hybridization times were 1 and $2 \mathrm{~h}$. In one case, a hybridization time of $4 \mathrm{~h}$ was additionally investigated. The effects of treatment on the cellular targets by ethanol-dehydration without and with RNase-treatment followed by pepsin digestion $(5,10,20,40$ min) were studied quantitatively. In all these cases, the painting signals were clearly visible by visual inspection; quantitatively, an increased fluorescence intensity of the painted area was measured by the CCD camera.

In Figure 2, a typical example of a CCD camera image is shown that also corresponds to the visual inspection. A 2-h hybridization time was chosen. Figure $2 \mathrm{a}$ represents the raw CCD image, while Figure $2 b$ shows the corresponding image after automatic global digital segmentation and discrimination. In this case, RNase A treatment and pepsin digestion were omitted.

To obtain a quantitative measure for the FISH efficiency, that is, an estimate for the signal-to-noise ratio in each metaphase spread, line scans and mean luminance scans were applied to the three brightest chromosomes (according to the mean chromosome intensity) (Figure 3). For each scan method, a FISH-efficiency factor was calculated. Figure 4 shows two different FISH-efficiency factors obtained from about 20 randomly chosen metaphase spreads for each type of experiment. As expected, the FISH-efficiency factors achieved by the line-scan procedure showed higher values than the ones obtained by the mean-luminance-scan procedure. However, the values derived from the mean luminance scan represent a more realistic result, since this procedure includes the measurement of 
the whole luminance of a labeled chromosome. Nevertheless, the values for both procedures indicate the same general tendency corresponding to the choosen FISH conditions. Only the line-scan data for one of the hybridization conditions $\left(2 \mathrm{~h} \mathrm{t}_{\mathrm{hyb}} / 20 \mathrm{~min}\right.$ pepsin digestion) lead to an overproportional value for the line scan FISH-efficiency factor as compared with the value obtained by the mean luminance scan.

Using the mean-luminance evaluation, no substantial difference in the FISH-efficiency factors was found for 1 or $2 \mathrm{~h}$ hybridization time after RNase/ pepsin treatment of 10 and $20 \mathrm{~min}$. The 1-h data set suggested a possible increase of the FISH-efficiency factor with increasing the pepsin exposure time to $40 \mathrm{~min}$. However, similar signal ratios were also obtained omitting the RNase/pepsin treatment and working with 2-h hybridization time. In contrast, so far, no satisfactory painting was obtained for 1-h hybridization time if RNase/pepsin treatment was omitted. For the 1-h hybridization condition, an RNase treatment with at least $5 \mathrm{~min}$ pepsin digestion produced better results. Finally, the highest FISH-efficiency factor observed in these experiments and based on the mean luminance scan was determined as about 3 (i.e., 300\% mean labeling intensity ratio) for 4-h Fast-FISH hybridization without RNase/pepsin treatment. In our experience, FISH-efficiency factors of 2 or 3 are completely sufficient to allow a clear identification of painted chromosomes, especially if digital microscopy and automated image analysis is used. This was true even in the case of FISH-efficiency factors of about 1.5.

\section{DISCUSSION}

In this report, it is shown, to our knowledge for the first time, that FastFISH of metaphase spreads obtained from human lymphocytes can be performed using repeat-depleted complex probes in the absence of suppressor DNA (Fast-Painting). Here, a repeatdepleted DNA library of the q-arm of the human chromosome 15 (3) was used. For the evaluation, digital fluorescence microscopy and quantitative image analysis were applied. The flexibil-
Table 1. Features of Fast-Painting Protocol

1. Allows working without DNA-denaturing chemical agents such as formamide, which has been classified as a toxic agent by the Swiss Drug Catalogue.

2. A substantial reduction of the workload and the related amount of chemical agents used, based on, for example, the number of working steps (no pretreatments required, no preannealing steps and only one washing step). This should contribute strongly to the robustness of the whole procedure, particularly in the case of large-scale applications of FISH (e.g., routine diagnostics and analysis).

3. Especially useful for routine (clinical) applications is the rapidness of the whole procedure. The protocol's very short hybridization time allows use of the same equipment (e.g., water baths, thermal cyclers) for more than one FISH experiment per day (typically 3 or 4 ).

4. If repeat-depleted complex libraries are used, a major economical advantage is possible due to the elimination of suppressor DNA (Cot-1 DNA) in the FISH experiment. To produce these libraries, repeat-depletion of the DNA-source probe in solution also requires high amounts of Cot-1 DNA. But in contrast to standard FISH protocols, once a complex probe has been repeat-depleted, it can be amplified and used further without consuming Cot-1 DNA.

5. An additional advantage of the use of repeat-depleted complex probes is that the fluorescence labeling of the probe is restricted to the low repetitive/unique sequences. Thus the stringency requirements are highly simplified.

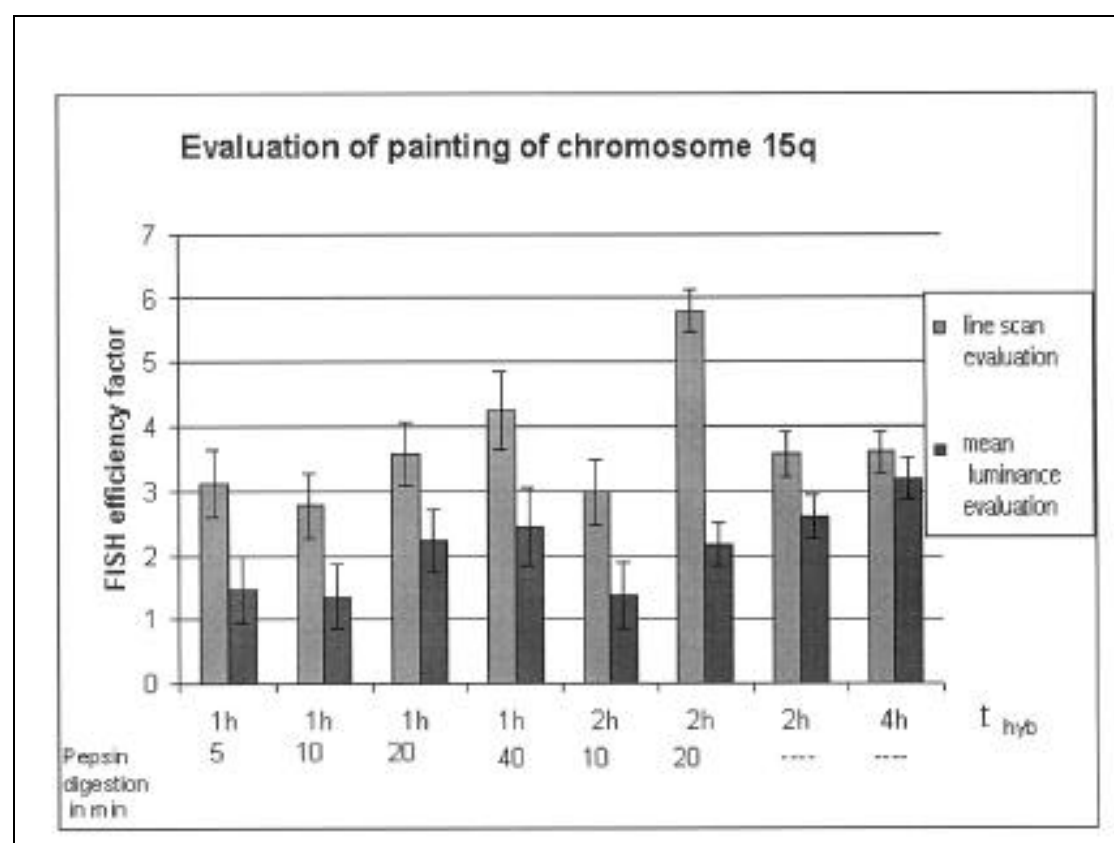

Figure 4. Mean FISH-efficiency factors of Fast-Painting of chromosome 15q arms obtained for different protocols. For line-scan evaluation (light gray, left column), the mean intensity ratios between the painted chromosome 15q arms and the brightest non-painted chromosome were used. For mean-luminance evaluation, the mean luminance ratios between the painted chromosome $15 \mathrm{q}$ arms and the brightest non-painted chromosome (dark gray, right column) were plotted. Every bar represents an average of 20 randomly chosen metaphase spreads. Besides the effect of variation of the hybridization time $\left(\mathrm{t}_{\text {hyb }}\right)$ from 1-4 h, also shown is the effect of pretreatment with RNase and pepsin (no treatment, 5-40 min pepsin digestion always accompanied by the same RNase digestion of $1 \mathrm{~h}$ ) on the mean FISH-efficiency factor. The error bars indicate the statistical error of the mean value. 
ity of the developed evaluation algorithm makes it potentially useful for chromosome aberration studies. The conditions for Fast-Painting were derived from the experience with FastFISH using repetitive DNA probes $(2,6,8,10,11)$. Analogous to those procedures, the complexity of painting protocols was considerably simplified.

Comparing the results presented here, the pretreatment of metaphase spreads by RNase/pepsin digestion appears to be unnecessary. However, since then, $2 \mathrm{~h}$ of hybridization were required to obtain reasonable painting results, omitting RNase/pepsin pretreatment did not contribute to a further acceleration of the process. Nevertheless, the absence of protease has the advantage that the procedure may be more gentle for the morphology of the target material. This might be seen as a first step to a more physiological FISH approach. As a further advantage of the Fast-FISH principle, recent results of Fast-FISH with a repetitive DNA probe indicated that there might be conditions that allow a complete omission of heat or formamide denaturation of the chromosomal target (5).

The new types of complex DNA probes applied here are highly suitable for Fast-FISH protocols since they significantly enlarge the range of application of this technique. In summary, the Fast-Painting protocol derived here offers the features described in Table 1.

In addition, the removal of repetitive sequences from complex probes should allow the straightforward simultaneous application of probes for chromosome site-specific, repetitive and non-repetitive DNA target sequences in FastFISH for multicolor labeling. This expectation has recently been experimentally realized (16).

\section{ACKNOWLEDGMENTS}

The authors gratefully acknowledge the financial support of the Deutsche Forschungsgemeinschaft and the German Bundesminister of Education, Science, Research and Technology. We thank W. Fischer for support in the development of image evaluation procedures. The support of Prof. T. Cremer, Munich, is gratefully acknowledged.

\section{REFERENCES}

1.Arakaki, D.T. and R.S. Sparkes. 1963. Microtechnique for culturing leukocytes from whole blood. Cytogenetics 2:57.

2.Celeda, D., K. Aldinger, F.-M. Haar, M. Hausmann, M. Durm, H. Ludwig and C. Cremer. 1994. Rapid fluorescence in situ hybridization with repetitive DNA probes: quantification by digital image analysis. Cytometry 17:13-25.

3.Craig, J.M., J. Kraus and T. Cremer. 1997. Removal of repetitive sequences from FISH probes using PCR-assisted affinity chromatography. Hum. Genet. 100:472-476.

4.Cremer, T., P. Lichter, J. Borden, D.C. Ward and L. Manuelidis. 1988. Detection of chromosome aberrations in metaphase and interphase tumor cells by in situ hybridization using chromosome-specific library probes. Hum. Genet. 80:235-246.

5.Durm, M., F.M. Haar, M. Hausmann, H. Ludwig and C. Cremer. 1997. Non-enzymatic low temperature fluorescence in situ hybridization of human chromosomes with a repetitive satellite III probe. Z. Naturforsch. [C] 51:82-88.

6.Durm, M., F.-M. Haar, M. Hausmann, H. Ludwig and C. Cremer. 1996. Optimization of fast-fluorescence in situ hybridization with repetitive $\alpha$-satellite probes. Z. Naturforsch. [C] 51:253-261.

7.Durm, M., M. Hausmann, K. Aldinger, H. Ludwig and C. Cremer. 1996. Painting of human chromosome 8 in fifteen minutes. Z Naturforsch. [C] 51:435-439.

8.Durm, M., I. Sorokine-Durm, F.M. Haar, M. Hausmann, H. Ludwig, P. Voisin and C. Cremer. 1998. Optimized FISH technique for rapid, simultaneous labelling of all human centromeres. Cytometry 31:153-162.

9.Guan, X.-Y., P.S. Meltzer and J.M. Trent. 1996. Rapid generation of whole chromosome painting probes (WCPs) by chromosome microdissection. Genomics 22:101-107.

10.Haar, F.-M., M. Durm, K. Aldinger, D. Celeda, M. Hausmann, H. Ludwig and C. Cremer. 1994. A rapid FISH technique for quantitative microscopy. BioTechniques 17:346-353.

11.Haar, F.-M., M. Durm, M. Hausmann, H. Ludwig and C. Cremer. 1996. Optimization of Fast-FISH for $\alpha$-satellite DNA probes. J. Biochem. Biophys. Methods 33:43-54.

12.Lichter, P. and T. Cremer. 1992. Chromosome analysis by non-isotopic in situ hybridization, p. 157-192. In D.E. Rooney and B.H. Czepulkowski (Eds.), Human Cytogenetics-A Practical Approach. IRL Press, Oxford.

13.Lichter, P., T. Cremer, J. Borden, L. Manuelidis and D.C. Ward. 1988. Delineation of individual human chromosomes in metaphase and interphase cells by in situ suppression hybridization using recombinant DNA libraries. Hum. Genet. 80:224-234.

14.Moorhead, P.S., P.C. Nowel, W.J. Mellham, B.M. Battips and D.A. Hungerford. 1960. Chromosome preparation of leucocytes cultered from human peripheral blood. Exp. Cell Res. 20:613-616.

15.Pinkel, D., J. Landegent, C. Collings, J.
Fuscoe, R. Segraves, J. Lucas and J.W. Gray. 1988. Fluorescence in situ hybridization with human chromosome specific libraries. Detection of trisomy 21 and translocations of chromsome 4. Proc. Natl. Acad. Sci. USA 85:9138-9142.

16.Schüßler, L., M. Durm, H. Münch, J. Craig, H. Ludwig, M. Hausmann and C. Cremer. 1997. Multicolor Fast-FISH of human metaphase spreads using a chromosome specific repeat depleted DNA library probe in combination with centromere probes. 10 . Heidelberger Zytometric Symp., ISSN 09495347:95.

Received 26 September 1997; accepted 29 December 1997.

Address correspondence to:

Dr. Christoph Cremer

Institut für Angewandte Physik

Albert-Überle-Str. 3-5

D-69120 Heidelberg, Germany

Internet: cremer@popeye.aphys2.uni-heidelberg.de 